\title{
A Marine Radar Wind Sensor
}

\author{
HEIKO DANKERT \\ GKSS Research Centre, Geesthacht, Germany, and Computational Vision Lab, California Institute of Technology, \\ Pasadena, California \\ JOCHEN HORSTMANN \\ GKSS Research Centre, Geesthacht, Germany
}

(Manuscript received 10 May 2006, in final form 8 November 2006)

\begin{abstract}
A new method for retrieving the wind vector from radar-image sequences is presented. This method, called WiRAR, uses a marine X-band radar to analyze the backscatter of the ocean surface in space and time with respect to surface winds. Wind direction is found using wind-induced streaks, which are very well aligned with the mean surface wind direction and have a typical spacing above $50 \mathrm{~m}$. Wind speeds are derived using a neural network by parameterizing the relationship between the wind vector and the normalized radar cross section (NRCS). To improve performance, it is also considered how the NRCS depends on sea state and atmospheric parameters such as air-sea temperature and humidity. Since the signal-tonoise ratio in the radar sequences is directly related to the significant wave height, this ratio is used to obtain sea state parameters. All radar datasets were acquired in the German Bight of the North Sea from the research platform FINO-I, which provides environmental data such as wind measurements at different heights, sea state, air-sea temperatures, humidity, and other meteorological and oceanographic parameters. The radar-image sequences were recorded by a marine X-band radar installed aboard FINO-I, which operates at grazing incidence and horizontal polarization in transmit and receive. For validation WiRAR is applied to the radar data and compared to the in situ wind measurements from FINO-I. The comparison of wind directions resulted in a correlation coefficient of 0.99 with a standard deviation of $12.8^{\circ}$, and that of wind speeds resulted in a correlation coefficient of 0.99 with a standard deviation of $0.41 \mathrm{~m} \mathrm{~s}^{-1}$. In contrast to traditional offshore wind sensors, the retrieval of the wind vector from the NRCS of the ocean surface makes the system independent of the sensors' motion and installation height as well as the effects due to platform-induced turbulence.
\end{abstract}

\section{Introduction}

This paper describes a radar-based remote sensing technique called WiRAR, which enables the measurement of the ocean surface wind from towers and ships. A marine radar operating at $\mathrm{X}$ band has the capability of measuring the backscatter from the ocean surface in space and time under most weather conditions and independent of lighting conditions. There are no biases due to wind sensor motion and height variations. Since the radar measures the wind from the ocean surface beside the platform, blockage and shadowing effects due to the sensor platform are also strongly reduced.

Corresponding author address: Heiko Dankert, Computational Vision Lab, California Institute of Technology, 1200 E. California Blvd., Pasadena, CA 91125.

E-mail: heiko@vision.caltech.edu
Marine-radar-image sequences have previously been used to measure two-dimensional wave spectra and significant wave heights (Borge et al. 1999) and wave groups (Dankert et al. 2003a). Image sequences of the ocean surface elevation may be extracted (Dankert and Rosenthal 2004; Borge et al. 2004; Dankert et al. 2005). Other such measurements include the mean nearsurface current (Senet et al. 2001), current fields, and bathymetry in inhomogeneous areas like coastal zones or areas with current gradients (Bell 1999; Trizna 2001; Dankert 2003). Finally, the motion of wind gusts has been studied (Dankert et al. 2004).

The frictional force of the wind field generates surface roughness that increases with wind speed (Lee et al. 1995; Trizna and Carlson 1996; Trizna 1997; Hatten et al. 1998). By exploiting this phenomenon, microwave radar can measure wind vectors near the surface of the

DOI: 10.1175/JTECH2083.1

(C) 2007 American Meteorological Society 
ocean. For radar backscatter at grazing incidence $\left(\geq 85^{\circ}\right)$, the normalized radar cross section (NRCS) is proportional to the spectral density of the surface roughness on scales comparable to the radar wavelength (Bragg scattering). In the case of $\mathrm{X}$ band at grazing incidence this is $\approx 1.5 \mathrm{~cm}$. In addition, at grazing incidence, radar backscatter is induced by other scattering mechanisms, for example, wedge scattering (Lyzenga et al. 1983) or small-scale wave breaking (Wetzel 1990; Askari et al. 1996; Trizna 1997). Long surface waves modulate small-scale surface roughness, which in turn modulates the radar backscatter. At grazing incidence the modulation stems from tilt and hydrodynamic modulation as well as geometrical shadowing of the radar beam due to the ocean waves (Wetzel 1990). These modulation mechanisms lead to the imaging of surface waves whose wavelengths are greater than 2 times the radar resolution. The modulation of the NRCS is mathematically described by the modulation transfer function (MTF), which is a sum of the four contributing processes: shadowing, tilt modulation, hydrodynamic modulation, and wind modulation. For a detailed description of scattering and modulation mechanisms at low grazing incidence, refer to a special issue of IEEE Transactions on Antennas and Propagation (1998, Vol. 46, No. 1).

The NRCS is typically largest when the wind blows directly toward the radar (upwind) and decreases to a minimum when the wind direction is orthogonal to the radar look direction (crosswind). Another smaller maximum in NRCS occurs when the wind blows directly away from the radar (downwind). However, for grazing incidence at $\mathrm{X}$ band with horizontal $(\mathrm{HH})$ polarization in transmit and receive the downwind maximum does not exist (Trizna and Carlson 1996; Dankert et al. 2003b).

The relation between the near-surface wind vector and NRCS can be described by a geophysical model function (GMF) of the form

$$
\sigma_{0}=a(\theta) \cdot u^{\gamma(\theta)}[1+b(u, \theta) \cos \Delta \Phi+c(u, \theta) \cos 2 \Delta \Phi],
$$

where $\sigma_{0}$ represents NRCS, $u$ represents wind speed, $\Delta \Phi$ represents the relative angle between the radar look and wind direction, and $\theta$ is the nadir incidence angle. The quantities $a, \gamma, b$, and $c$ are empirical parameters that are typically determined by measured data. In case of $\mathrm{HH}$ polarization at grazing incidence the coefficient $c=0$. The single peak stands in contrast to radar measurements of the sea surface in $\mathrm{X}$ band with VV polarization and radars operating at moderate incidence angles where two maxima are observed, one upwind and one downwind (Trizna and Carlson 1996; Hatten et al. 1998). Local minima are crosswind, and the upwind NRCS is slightly higher than the downwind one. The single peak makes radar measurements obtained with $\mathrm{HH}$ polarization at grazing incidence the best choice for wind direction measurements, because they enable the retrieval of unambiguous wind directions. Equation (1) shows that $\sigma_{0}$ is an exponential function of wind speed and a harmonic function of its direction. Note that a specific NRCS value cannot be associated with a unique wind speed and direction pair. However, if wind direction is known a priori, it is possible to estimate wind speed.

The conventional approach using Eq. (1) is only applicable if the ocean surface is imaged over the full azimuth to enable covering of the required upwind peak. This is often not the case. Using the upwind peak for a direct measurement of the wind direction is further not very accurate and provides no information about the local wind field. In addition, the turbulence due to the sensor platform contaminates the downwind measurements. For these reasons, WiRAR uses a different method to determine the wind vector.

The WiRAR algorithm consists of two parts. In the first part, local wind directions are retrieved from the wind-induced streaks that are visible in radar-image sequences. These streaks are aligned with the mean wind direction. The streaks are typically imaged by the radar at scales of approximately $50-500 \mathrm{~m}$ and are extracted by a method based on derivation of local gradients [local gradient method (LGM)] (Horstmann et al. 2002; Koch 2004; Dankert et al. 2003b). In the second part, the wind speed is derived from the NRCS, which is strongly dependent on the ocean surface wind speed. This dependency is parameterized using a neural network (NN). NNs have already been successfully applied for satellite-based wind retrieval, for example, from spaceborne scatterometers (Richaume et al. 2000) and synthetic aperture radar (SAR) images (Horstmann et al. 2003, 2005).

Several studies have already analyzed the wind dependency of radar data acquired at gazing incidence (Chaudhry and Moore 1984; Lee et al. 1996; Hatten et al. 1998; Dankert et al. 2003b). In these studies the dependencies of the NRCS on both wind speed and direction have been investigated. Keller et al. (1985, 1994) have studied the dependency of the NRCS on wind speed, atmospheric stability, and sea state. They found that under unstable conditions the NRCS is higher than in near-neutral conditions, while the oceanwave radar MTF is lower. In near-neutral atmospheric conditions the MTF decreases with long-wave slope, while the NRCS increases. 
Wind speed estimates are further refined by taking the dependency of sea surface friction on the sea state and atmospheric conditions into account. The sea state is described by the wave phase speed at the spectral peak $c_{p}$ as well as the ratio between the signal of the linear surface gravity waves and the background noise [signal-to-noise ratio (SNR)]. Practice has shown that the square root of the SNR is proportional to the significant wave height of the observed wave field (Ziemer 1991). The SNR is independent of the wind field. Both parameters, $c_{p}$ and SNR, are extracted from the radarimage sequences. Considering all information available from radar-image sequences makes a marine radar system an accurate stand-alone wind sensor. The atmospheric conditions are characterized by the air-sea temperature difference $\Delta \vartheta_{(a, s)}$ and the air humidity $q_{r}$. This additional information further improves the accuracy of the radar wind measurements.

The investigated radar-image sequences were recorded by the Wave Monitoring System (WaMoS II), developed at the GKSS Research Centre (Geesthacht, Germany). For validation of WiRAR, wind vectors from 4786 radar-image sequences acquired at the FINO-I platform are compared to the in situ measurements recorded aboard FINO-I.

The paper is organized as follows. In section 2 the radar system and available in situ data are presented. In section 3 the wind direction retrieval algorithm is introduced and validated by comparison to in situ data recorded at the radar platform. In section $4, \mathrm{NNs}$ are introduced and applied to wind speed retrieval from the radar-image sequences. The dependency of the NRCS on sea state and atmospheric conditions is shown and considered in the neural network's estimate of the GMF. Finally, our wind speed estimates are compared to the in situ measurements collected at FINO-I. Conclusions and an outlook are given in section 5.

\section{Investigated data}

The radar utilized in this paper is a commercial marine radar (Furuno FR-2125-B) with peak power output of $25 \mathrm{~kW}$ operating at $9.5 \mathrm{GHz}$ (X band) near grazing incidence. The 8 -ft $(2.4 \mathrm{~m})$ open array antenna (type $\mathrm{XN} 24 \mathrm{AF} / 8$ ) is horizontally polarized with a $0.95^{\circ}$ horizontal beamwidth. The radar antenna rotates with period of $2.5 \mathrm{~s}(24 \mathrm{rpm})$. At a distance of $\approx 750 \mathrm{~m}$ the spatial resolution is $\approx 10 \mathrm{~m}$ in range and $\approx 12 \mathrm{~m}$ in azimuth. All data were acquired in the near-range mode, where the radar covers an area within a radius of $\approx 2000$ $\mathrm{m}$. The datasets were recorded by WaMoS II, which enables digitizing time series of radar sea clutter images on an operational basis. Marine radar systems are equipped with a logarithmic amplifier, and the received signal is not radiometrically calibrated. The backscattered signal of each radar resolution cell is digitized with 8-bit precision. Each of the radar-image sequences investigated here consists of 32 images representing $\approx 80 \mathrm{~s}$. They cover a period from August 2003 until November 2004, representing 4786 acquisition times with wind speeds of up to $16 \mathrm{~m} \mathrm{~s}^{-1}$. All data were acquired in the German Bight of the southern North Sea from the research platform FINO-I, which is located at $54^{\circ} \mathrm{N}, 6.6^{\circ} \mathrm{E}$ in a water depth of $\approx 30 \mathrm{~m}$.

The radar antenna is installed at a height of $20 \mathrm{~m}$ above mean sea level. The investigated area covers ranges between 600 and $2100 \mathrm{~m}$, corresponding to grazing incidence angles between $88.1^{\circ}$ and $89.5^{\circ}$. With the antenna's vertical directivity of $20^{\circ}$, even ship motions in severe sea state are compensated.

Figure 1 shows the research platform FINO-I with its 100-m-long mast. The radar antenna is mounted just below the helideck at the northern part of the platform. The radar-image sequence depicts a wave field propagating in an easterly direction. The southern quadrant of the images contain radar shadows originating from the platform equipment, which have been masked (black sector) and which were excluded from the investigations.

At FINO-I, meteorological and oceanographic parameters are measured at various heights and depths. The instrumentation of the met mast consists of cup anemometers and wind vanes in heights ranging from 30 to $100 \mathrm{~m}$ above mean sea level approximately every $10 \mathrm{~m}$, which are stored as 10-min averages. The cup anemometers are supplemented by ultrasonic anemometers at 40-, 60-, and 80-m height, which are sampled at $10 \mathrm{~Hz}$. In this investigation only wind measurements at 30-m height were utilized. The air temperature was measured with modified PT100 sensors at five different levels and the sea surface temperature at $3 \mathrm{~m}$ below mean sea level. The air humidity was measured with classical hair hygrometers at 33-, 50-, and 100-m heights. Last but not least, the sea state, in particular significant wave height and peak period, was measured by a Wavec buoy located at a distance of 300-400 m off the platform as well as by WaMoSII (Borge et al. 1999).

\section{Measuring wind direction}

The WiRAR wind direction retrieval is based on the imaging of linear features aligned along the wind direction. Most of these features are associated with wind streaks (Drobinski and Foster 2003) or streaks from 


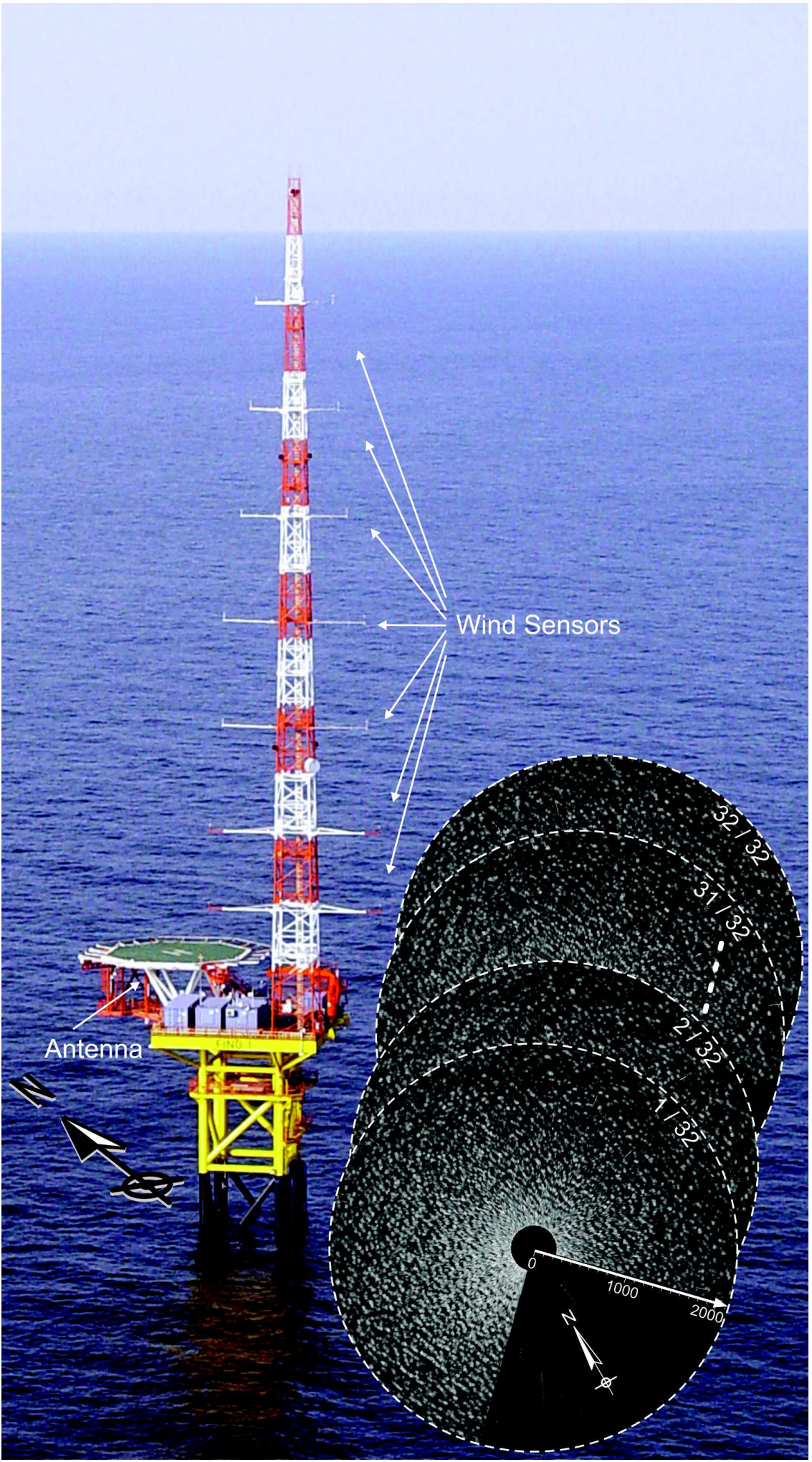

FIG. 1. WaMoS system installed on the research platform FINO-I in the German Bight. Various atmospheric sensors are mounted on the measurement mast and at the framework of the complex. 


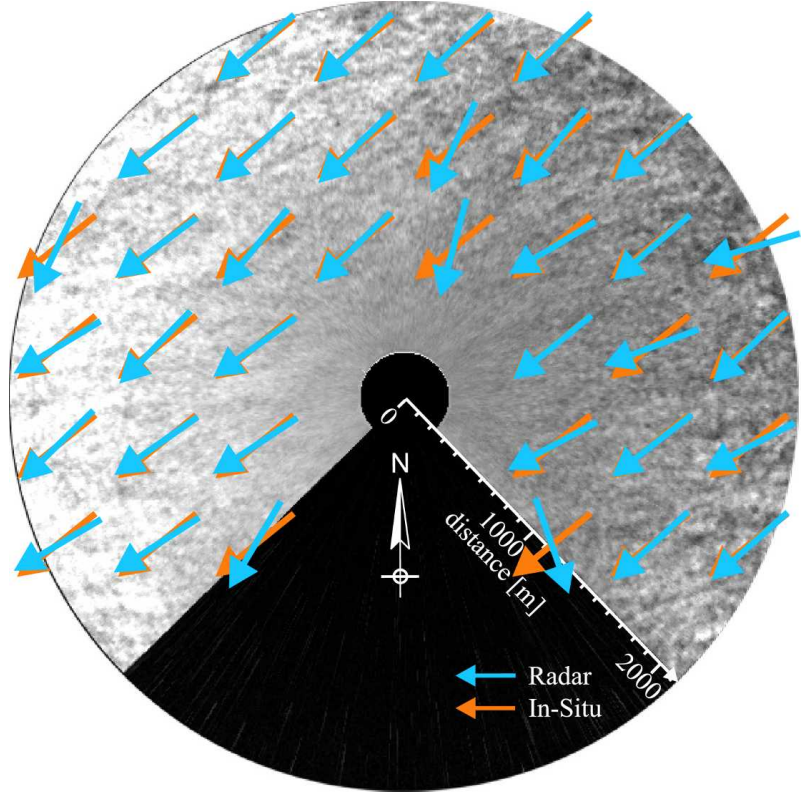

FIG. 2. Local wind directions at the ocean surface retrieved from the mean NRCS of a radar-image sequence of 32 images taken at FINO-I on $2 \mathrm{Feb} 2004$. The global in situ wind direction was $52^{\circ}$ and the wind speed $11.6 \mathrm{~m} \mathrm{~s}^{-1}$. A black sector of $90^{\circ}$ in the southern direction is not considered.

foam or surfactants, which are visible in the radar images at scales between 50 and $500 \mathrm{~m}$ (Fig. 2). These streaks are also visible in SAR images at similar and larger scales and have been shown to be well aligned with the mean surface wind direction (Horstmann and Koch 2005). We compute the orientation of these linear features using the LGM, which has been used successfully for estimating SAR wind direction (Horstmann et al. 2002; Koch 2004).

In a first step, to separate wind from other signatures and to reduce the effect of speckle, a radar-image sequence is integrated over time (typically, 32 images representing $80 \mathrm{~s}$ of data). This removes patterns that are highly variable in the temporal domain such as ocean surface waves. Only static patterns such as the shadows and wind signatures remain visible in the integrated radar image.

In the next step, the integrated radar image is sequentially smoothed and reduced to resolutions of 20 , 40 , and $80 \mathrm{~m}$. The resulting three radar images retain spatial scales greater than 40,80 , and $160 \mathrm{~m}$. From each of these images, local orientations are computed using the normal to the local gradient (to within a $180^{\circ}$ ambiguity). From all of the retrieved orientations, only the most frequent orientations in a predefined area are selected. These resulting wind orientations typically vary by only a few degrees, except for cases where additional features are present in the radar image, for example, artifacts arising from bathymetry or current shear. In such cases the artificial structures are interpreted as wind streaks, which is avoided by identifying the artifacts before applying the LGM (Koch 2004).

The $180^{\circ}$ ambiguities can be removed in one of two ways. Wind gusts become visible in radar-image sequences after filtering. The motion of these gusts can be analyzed using an opticalflow-based motion estimation technique [Dankert et al. (2004)]. The more standard alternative technique is to estimate the shift of moving image patterns between two datasets by computing the cross-correlation function (CCF). In the latter method, the respective propagation direction is indicated by the location of the CCF peak. Alternatively, the shift can also be estimated by looking at the cross-spectrum (CS), which is defined as the Fourier spectrum of the CCF. In the CS the shift of the different harmonic features in the image is given by the respective phases of the complex valued CS. The directions resulting from the CS are always within $90^{\circ}$ of the in situ measured wind direction, thus resolving the $180^{\circ}$ directional ambiguity. Last but not least, the directional ambiguity can be removed using the dependency of the NRCS on the antenna look direction, because for X-band radars operating at grazing incidence with $\mathrm{HH}$ polarization a peak exists only in the upwind direction (Trizna and Carlson 1996; Dankert et al. 2003b). This method is only applicable if the ocean surface is imaged over the full azimuth, which is often not the case. In addition to this restriction, using the upwind peak for a direct measurement of the wind direction is less accurate than the LGM.

In Fig. 2, the resulting local mean directions are plotted for a sample scale (blue arrows). It can be seen that they agree well with the wind direction measured at the radar platform at a height of about $30 \mathrm{~m}$ (red arrows).

For validation of the WiRAR wind direction retrieval method the radar-retrieved mean wind directions of 4786 radar-image sequences are compared to the in situ measurements of the FINO-I platform, which were acquired at a height of $30 \mathrm{~m}$. Figure 3 gives the comparison with the following main statistical parameters: correlation coefficient of 0.99 , bias of $0.3^{\circ}$, and standard deviation of $12.8^{\circ}$. The bias might be expected on the basis that an Ekman spiral is resolved by averaging over all events.

Figure 4 shows bias (solid line) and standard deviation (dotted line) of radar and in situ wind direction difference, plotted against wind speed. The dependence of the bias on wind speed is negligible. However, the standard deviation decreases significantly with increasing wind speed as the wind streaks become more pro- 


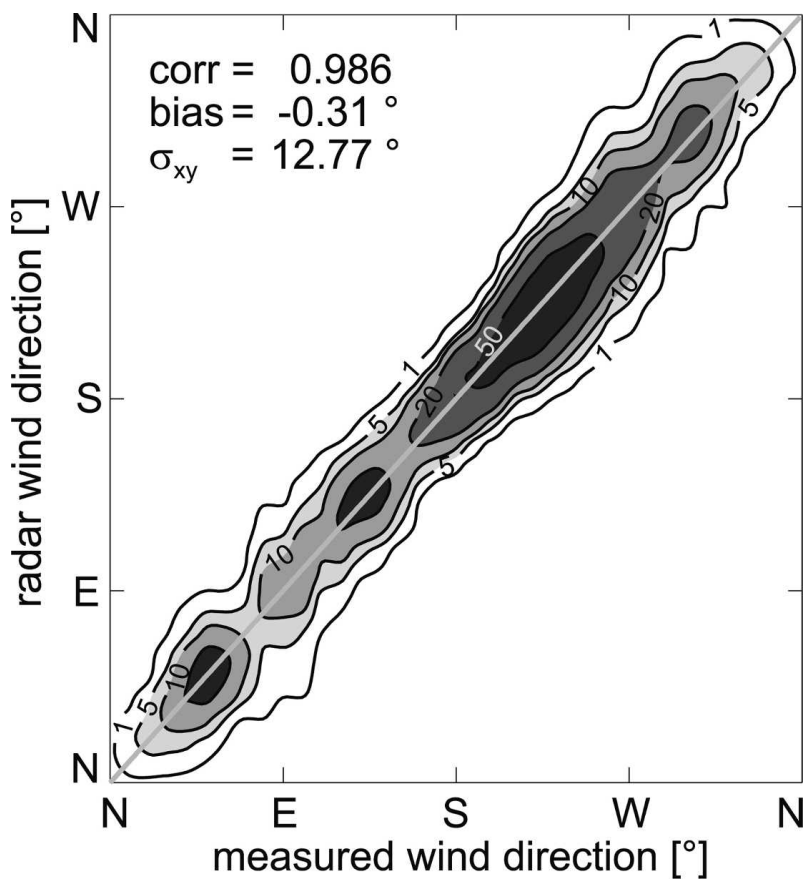

FIG. 3. Scatterplot of in situ and radar-retrieved wind directions (4786 datasets).

nounced. Under weak wind conditions the NRCS is less indicative for atmospheric than oceanic processes, such as bathymetric effects and current shear. In such cases, the wind directionality of the NRCS is dominated by wind-driven ripple waves and the single peak in the upwind direction at $\mathrm{HH}$ polarization and grazing incidence.

\section{Measuring wind speed}

The WiRAR wind speed retrieval is based on the dependence of the NRCS on the local wind vector. This

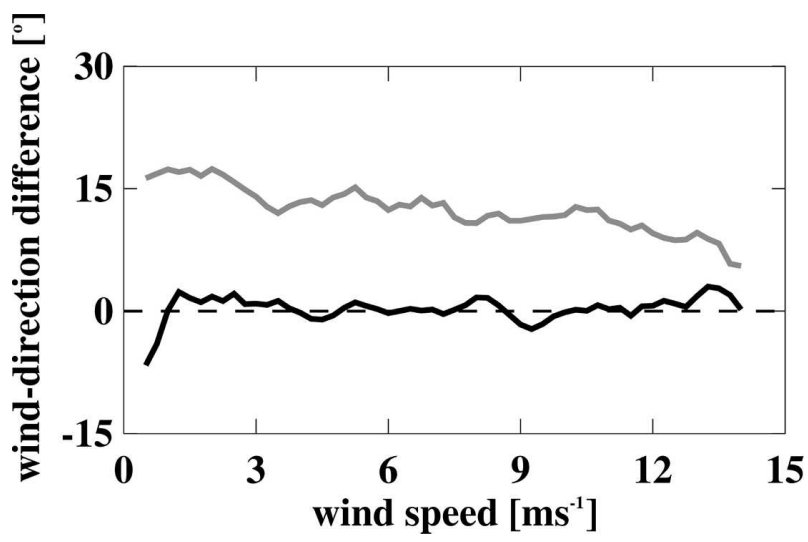

FIG. 4. Bias (black line) and standard deviation (gray line) of difference between radar and in situ wind directions vs wind speed. dependency is described by a GMF [Eq. (1)], which in the following is developed using NNs. The NNs do not require explicit models for the radar-imaging process and can therefore be easily applied to any system configuration (e.g., polarization, incidence angle, etc.). The only requirements for application of an $\mathrm{NN}$ to radar wind speed retrieval is the relative radiometric stability of the system, which is the case for typical marine radars, such as the one used in the FINO-I setup. Unfortunately, marine radar systems are normally not radiometrically calibrated. An NN should therefore be trained individually for every radar system. Suppose the relative response of all radars is similar; for example, their wind speed dependence is within a simple relationship. An easy calibration procedure could then be developed. In this case the calibration phase would consist only of a few measurements. This assumption is not the subject of this paper and needs further investigation. In the following a feed-forward backpropagation NN (available online at http://gfesun1. gkss.de/software/ffbp) is used as a multiple-nonlinearregression technique to parameterize the relationship between the radar intensity and ocean surface wind.

An NN is built up of several layers: an input layer, one or more hidden layers, and one output layer. Each layer consists of "neurons"; the input layer has as many neurons as input parameters, and the output layer has many neurons as output parameters. The number of hidden layers and the number of neurons in the hidden layer(s) depends on the problem. Each neuron in a layer is linked to each neuron of the neighboring layer with a weight. The output value $N_{\text {out }}$ of each neuron is derived according to

$$
N_{\text {out }}=S\left(-N_{\text {bias }}+\sum_{i=1}^{n} w_{i} x_{i}\right)
$$

where $N_{\text {bias }}$ is a bias value specific to each neuron, $n$ is the number of incoming links, $w$ is a weight specific to each neuron, and $x$ is the output value of the neuron in the preceding layer; $S$ is a nonlinear function assuming monotonically increasing values between 0 and 1 as the value of the argument goes from $-\infty$ to $\infty$. The most common nonlinear function, also used in the NNs applied here, is the Sigmoid function $\left(1+e^{-x}\right)^{-1}$. An NN operates sequentially from layer to layer; output neurons of the first layer are given by the input values. The output of each neuron of the first hidden layer is computed by the summation of the weighted inputs, shifting by the bias and application of the nonlinear function. This is repeated for each layer until the output layer is reached, giving the results of the NN. To determine an $\mathrm{NN}$, a sufficiently large set of input and output vectors 
TABLE 1. Schematic setup of an NN to determine a GMF for wind speed retrieval.

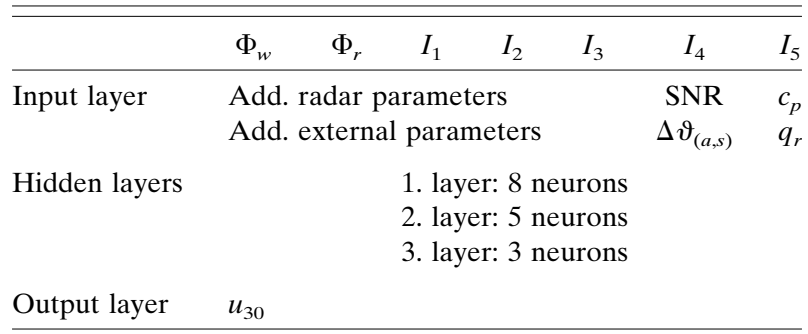

has to be available. During the training of the NN, the values of the biases and weights are changed to minimize the error function. The resulting trained $\mathrm{NN}$ has to be tested with test datasets for its generalization power, for example, whether reasonable results are produced for input values that are not included in the training sample. For training of the NN, the dataset that consisted of 4786 radar-image sequences was subdivided into a training dataset and a test dataset with a ratio of 2:1.

As discussed in Dankert et al. (2003b), there is a strong range-dependency of the mean NRCS (integrated over time), where the NRCS decreases with increasing distance from the radar antenna. Due to the very limited analog-to-digital converter ( 8 bit) of the utilized radar system, saturation can occur for higher wind speeds, especially in the near range (600-900 m), where the sensitivity of the NRCS on wind speed is higher at low wind speeds, while at high winds saturation occurs. In the far range (1800-2100 m) the sensitivity is larger for high wind speeds, whereas at low wind speeds the wind signal cannot be separated from the background noise. Therefore, utilization of the mean NRCS from the different range sectors enables a significantly better parameterization for the entire range of wind speeds. However, at far ranges the falloff of the NRCS depends on the refractivity profile. This dependency needs to be investigated in further studies.

To include the dependencies of NRCS on wind direction and range distance, each mean NRCS image (image sequence integrated over time) is subdivided into several range and azimuth bins. In our study, each radar image is divided into subareas of five $300-\mathrm{m}$ range intervals starting at a distance of $600 \mathrm{~m}$ and in azimuth sectors of $5^{\circ}$. For every subarea the mean $\operatorname{NRCS}\left(I_{1}-I_{5}\right)$ is determined.

\section{a. Consideration of the NRCS}

A schematic setup of the complete NN construction is shown in Table 1. In a first step, following Dankert et al. (2003b), NNs were trained using radar-measured wind direction $\Phi_{w}$, the mean radar intensities of the five

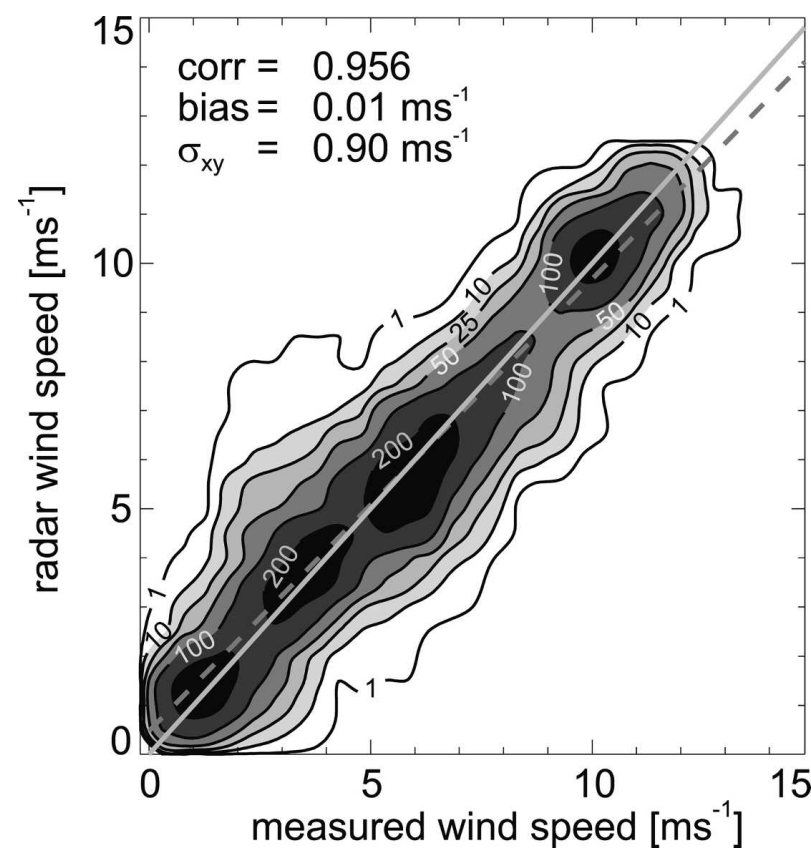

FIG. 5. Comparison of the wind anemometer wind speeds $u_{30}$ (mean of $10 \mathrm{~min}$ ) vs wind speeds retrieved from collocated marine radar images. The radar wind speeds were retrieved using an NN with the intensity of the mean radar image, antenna look direction, and the wind direction from the collocated anemometer data as input (4786 datasets).

range-azimuth cells in both crosswind directions, and the corresponding antenna look direction $\Phi_{r}$. There are three hidden layers with eight neurons in the first, five in the second, and three in the third hidden layer. The output layer consists of one neuron given by the wind speed $u_{30}$, measured at $30-\mathrm{m}$ height. The additional radar parameters and parameters from external sensors as input for the $\mathrm{NN}$ are explained in the following sections.

The crosswind directions are taken due to their higher wind sensitivity. Furthermore, artifacts caused by the platform due to wind shadowing or blockage are imaged in the downwind or up-wind direction, respectively. The crosswind directions are not influenced.

Figure 5 shows a scatterplot of in situ wind speeds $u_{30}$ versus radar-retrieved wind speeds using the resulting NN. Varying crosswind directions within $\pm 15^{\circ}$ of the true crosswind direction were used with the GMF. This results in a correlation coefficient of 0.96 with a bias of $0.01 \mathrm{~m} \mathrm{~s}^{-1}$ and a standard deviation of $0.90 \mathrm{~m} \mathrm{~s}^{-1}$. Considering only the true crosswind direction slightly decreases the standard deviation to $0.89 \mathrm{~m} \mathrm{~s}^{-1}$. Therefore, an accuracy of $\pm 15^{\circ}$ for wind direction measurements is sufficient for an accurate wind speed retrieval. In practice, the resulting parameterization can be used to estimate wind speeds as low as $\approx 1.0 \mathrm{~m} \mathrm{~s}^{-1}$. Physically the 
minimum wind speed for wind-wave generation is $0.7 \mathrm{~m} \mathrm{~s}^{-1}$.

To improve the given GMF for wind speed determination, the dependencies of the NRCS on additional parameters such as sea state and atmospheric conditions have to be considered. Information on the sea state can be extracted from the radar datasets, while atmospheric stratification conditions, such as the airsea temperature difference and the relative air humidity, need to be measured by external sensors.

\section{b. Consideration of the sea state}

The surface roughness over the water is primarily dependent on the surface wind speed and therefore on the vertical profile, which is dependent on sea state and stratification of the atmosphere. Physical mechanisms have been proposed accounting for the dependency of surface stress on sea state (Geenaert 1990).

Drennan et al. (2003) found the following roughnesswave relationship for developing wind seas $\left(u_{\star} / c_{p}>\right.$ $0.05)$ with unimodal spectrum:

$$
z_{0} / \sigma=a\left(u_{\star} / c_{p}\right)^{b},
$$

where $u_{\star}$ is the friction velocity; $c_{p}$ the phase speed of the waves at the spectral peak (Stewart 1974; Komen and Oost 1998; Donelan 1990); $\sigma$ is introduced as rms surface elevation; the significant wave height, $H_{S}=4 \sigma$; $z_{0}$ is the roughness parameter (Phillips 1977); and $a$ and $b$ are constants depending on the development of wind sea.

For the purpose of implementing the additional sea state dependency on the sea surface stress, represented by $H_{S}$ (beside $c_{p}$ ), into a wind speed parameterization from the NRCS it is sufficient to use the windindependent SNR (Ziemer 1991; Izquierdo et al. 2004). The dependency between SNR and sea state is described below. The radar system is still able to run as a stand-alone instrument for retrieving surface winds.

The NRCS is modulated by surface gravity waves and a noise component. Both components are statistically independent. Sea state modulation and noise component cannot be separated in the spatiotemporal domain. Using a three-dimensional fast Fourier transform (3D FFT), an image sequence $\mathrm{G}(\mathbf{r}, t)$ with location $\mathbf{r}=$ $(x, y)$ and time $t$ is transformed into wavenumberfrequency domain:

$$
I(\mathbf{k}, \omega)=|\operatorname{FFT}[G(\mathbf{r}, t)]|^{2},
$$

with wavenumbers $\mathbf{k}=\left(k_{x}, k_{y}\right)$ and angular frequencies $\omega$. The result is a $3 \mathrm{D}$-image power spectrum $I(\mathbf{k}, \omega)$. For each of the 4786 radar datasets the 3D-image power spectrum is determined. In the spectral domain, wavenumbers and frequencies of the imaged waves are con- nected by the dispersion relation of linear surface gravity waves as follows:

$$
\varpi\left(\mathbf{k} ; d, \mathbf{u}_{e}\right)= \pm \sqrt{g k \cdot \tanh (k d)}+\mathbf{k} \cdot \mathbf{u}_{e},
$$

where $\varpi$ is the intrinsic frequency, $g$ is the gravitational acceleration, $k$ the modulus of the wavenumber vector $\mathbf{k} d$ the water depth, and $\mathbf{u}_{e}$ the velocity of encounter between the water surface and the radar (Stoker 1957, 109-133).

After determining water depth $(\approx 30 \mathrm{~m}$ at FINO-I $)$ and velocity of encounter (between fixed platform and near-surface current vector), by fitting the theoretical dispersion relation to the signal coordinates of the linear surface waves in the wavenumber frequency domain (Young et al. 1985; Senet et al. 2001), the dispersion relation is used as a signal filter in the image power spectrum to separate the linear sea state signal from the background noise (Ziemer 1991):

$$
I_{\text {sig }}(\mathbf{k}, \omega)=I(\mathbf{k}, \omega) \delta\left[\omega-\varpi\left(\mathbf{k} ; d, \mathbf{u}_{e}\right)\right],
$$

where the delta function $\delta(\omega-\varpi)$ is the spectral bandpass filter with the dispersion relation $\varpi$. Figure 6 shows an example slice through a 3D-image power spectrum. There are clearly structures visible in the spectrum that are related to the signal of the linear surface gravity waves. Different from single satellite images, a $180^{\circ}$ unambiguous wavenumber image spectrum is determined by integrating over the positive frequencies of the signal-filtered image power spectrum.

The power of the background noise $I_{\text {noi }}$ is estimated by subtracting the power of the linear sea state $I_{\text {sig }}$ from the total power $I$ :

$$
I_{\text {noi }}(\mathbf{k}, \omega)=I(\mathbf{k}, \omega)-I_{\text {sig }}(\mathbf{k}, \omega) .
$$

With $I_{\text {sig }}$ and $I_{\text {noi }}$ the SNR is defined as

$$
\mathrm{SNR}=\frac{\int I_{\mathrm{sig}}(\mathbf{k}, \omega) M(k) d \mathbf{k} d \omega}{\int I_{\text {noi }}(\mathbf{k}, \omega) d \mathbf{k} d \omega} \forall k, \omega \neq 0 .
$$

The MTF $M(k) \sim k^{-\beta}$ is applied in order to correct for imaging effects (Plant 1989). The exponent was empirically determined with $\beta=1.2$ (Borge et al. 2004).

The SNR is independent of the wind and directly related to $H_{S}$ (Alpers and Hasselmann 1982):

$$
H_{S} \sim \sqrt{\mathrm{SNR}}
$$

whereas for determination of $H_{S}$, calibration constants need to be estimated by comparing radar and collocated buoy data. Figure 7 gives a comparison of the square root of determined SNR values from radar datasets and measured collocated buoy $H_{S}$. Measurements were partially available for the given radar datasets. With a correlation coefficient of 0.67 a typical good relationship between both parameters is given.

After separating wave signal from background noise, the SNR is determined for each radar dataset. In addi- 


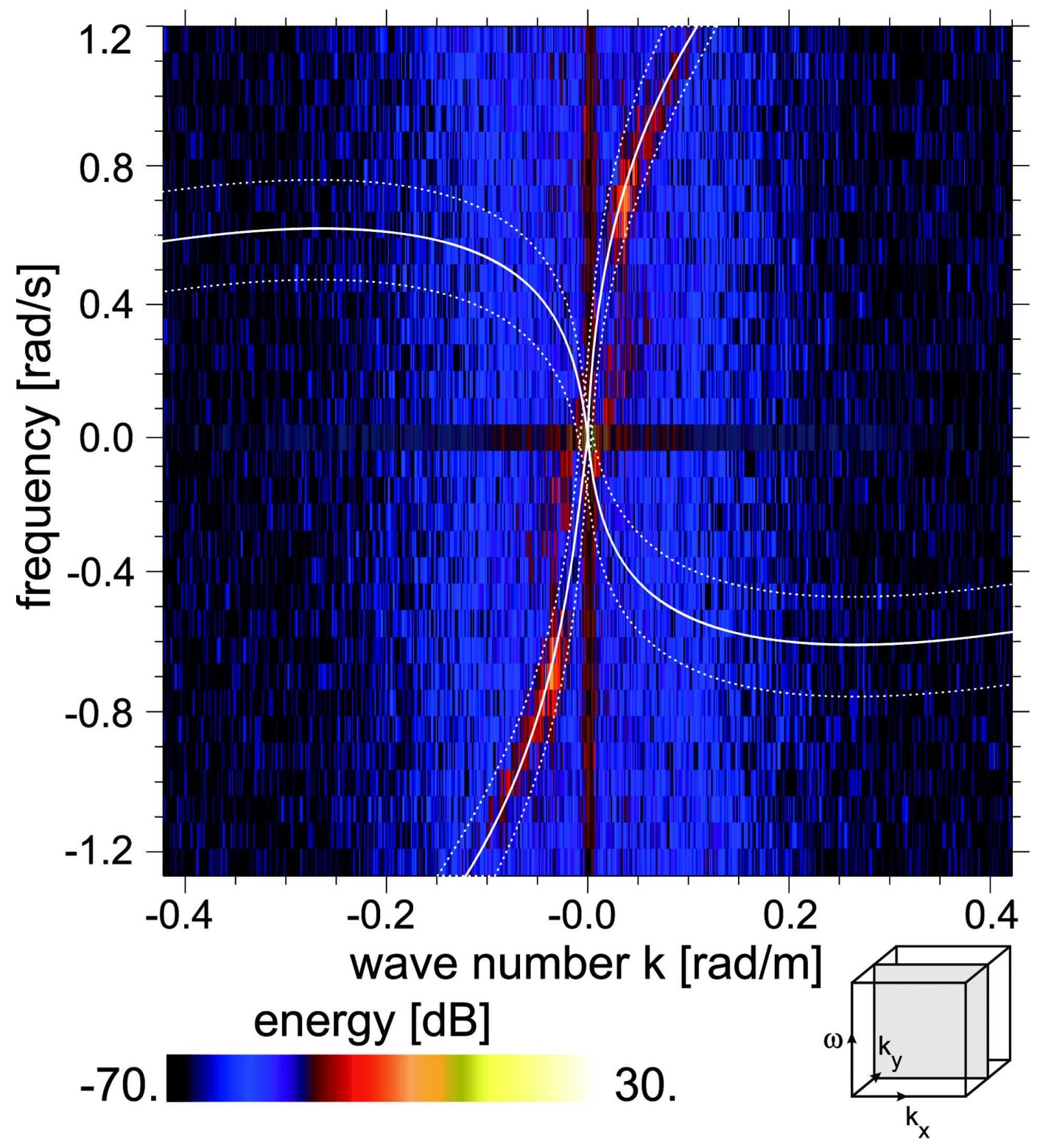

FIG. 6. Wavenumber-frequency slice through the 3D wavenumber-frequency power spectrum of a time series of 32 radar images. The modulation signal of the sea state is significantly imaged in its linear parts and located on the dispersion shell (solid curve), which spans the dispersion filter for signal and noise separation (area between dotted curves).

tion the phase speed of the peak wave component of the spectrum $c_{p}=\omega_{p} / k_{p}$, with peak wavenumber $k_{p}$ and peak frequency $\omega_{p}$, is computed from the positive frequency part of the signal-filtered power spectrum $I_{\text {sig }}^{+}(\mathbf{k}, \omega)$.

Both parameters, SNR and $c_{p}$, are analyzed for their influence on the wind parameterization. The same NN construction already used in section $4 \mathrm{a}$ is applied, and the SNR is taken as an additional input parameter. The resulting NN achieved a correlation coefficient of
0.97 , with a bias of $0.0 \mathrm{~m} \mathrm{~s}^{-1}$ and a standard deviation of $0.76 \mathrm{~m} \mathrm{~s}^{-1}$.

Before taking $c_{p}$ into account the SNR is analyzed for a dominating sea state signal. For low-wind cases with a low SNR there are no modulating wind waves, and $c_{p}$ cannot be determined. For a GMF considering $c_{p}$, such low-wind situations have to be excluded. Training an NN using the intensities of the mean radar image, the antenna look direction, the wind direction from the collocated anemometer, and $c_{p}$ results in an $\mathrm{NN}$ that 


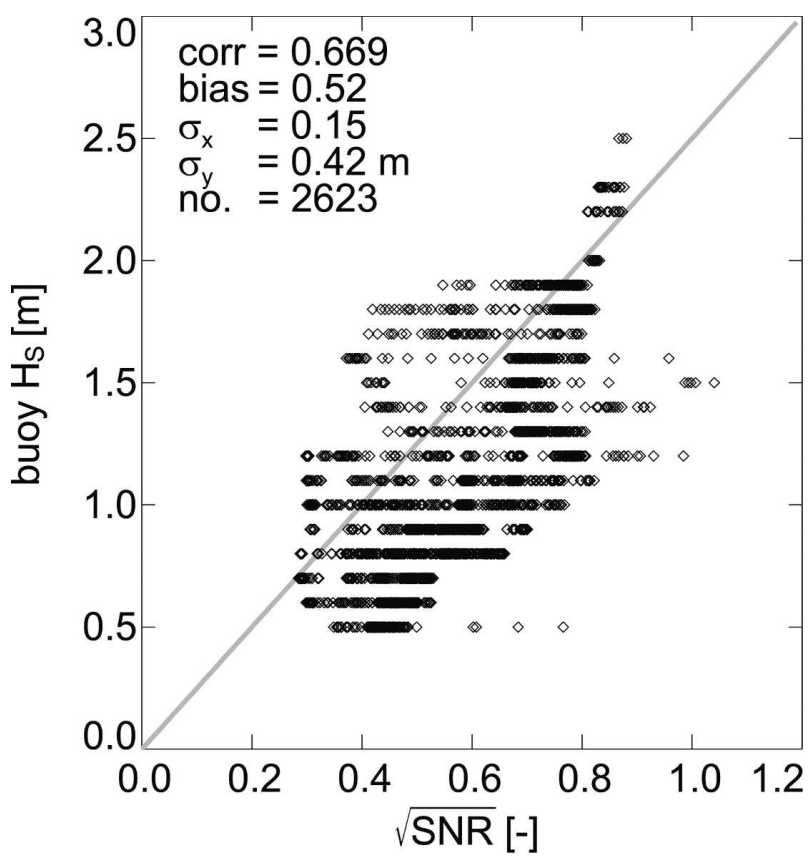

FIG. 7. Scatterplot of $\sqrt{\mathrm{SNR}}$ and buoy $H_{S}$ for the given radar datasets where buoy measurements were available (2623 datasets).

achieves a correlation coefficient of 0.96 , a bias of $0.0 \mathrm{~m} \mathrm{~s}^{-1}$, and a standard deviation of $0.86 \mathrm{~m} \mathrm{~s}^{-1}$ when compared to the in situ measurements.

Taking the SNR into account improves the correlation coefficient to 0.97 with a standard deviation of $0.66 \mathrm{~m} \mathrm{~s}^{-1}$. This parameterization can measure wind speeds below $1 \mathrm{~m} \mathrm{~s}^{-1}$. This is the most accurate measurement that can be obtained from marine radar without considering any external sensors.

\section{c. Consideration of the atmospheric conditions}

The stratification conditions in the lower marine atmospheric boundary layer (MABL), mainly denoted by the air-sea temperature difference, affect the stability of the air-sea interface and therefore also the wind profile. In turn, the wind profile influences the radar backscatter of the ocean surface. This leads at equivalent wind speeds in 10-m height to a higher NRCS at the sea surface in the case of an unstable MABL than for neutral and stable conditions (Keller et al. 1989).

Again an NN is trained considering additionally the air and sea temperature differences, which were measured by external in situ sensors. The main statistical parameters improve compared to the previously trained NNs, resulting in a correlation coefficient of 0.98 , a negligible bias, and a standard deviation of $0.61 \mathrm{~m} \mathrm{~s}^{-1}$. This parameterization can be used to estimate wind speeds as low as $\approx 0.75 \mathrm{~m} \mathrm{~s}^{-1}$.
The dependency between air temperature $\vartheta_{\text {air }}$, and relative air humidity $q_{r}$ is denoted

$$
q_{r}=q / q_{s}\left(\vartheta_{\text {air }}\right),
$$

where $q$ is the absolute or specific humidity, and $q_{s}$ is saturation specific humidity. The air density is closely related to relative humidity (or specific humidity, but the relative humidity is the measured value at FINO-I). A water molecule weight only $62.2 \%$ as much as the average dry air molecule. If dry air at a certain pressure and temperature has a density $\rho_{a, d}$, moist air of specific humidity $q$ has the same number of molecules per unit volume, but the density per unit volume of moist air is

$$
\rho_{a, m}=\rho_{a, d}(1+q) /(1+1.608 q) .
$$

With increasing relative humidity the density of moist air decreases. This results in a decreasing friction or small-scale roughness at the sea surface.

As a fourth investigative step, the dependency of relative air humidity $q_{r}$ on the small-scale roughness and therefore the NRCS is additionally considered in the parameterization for the radar wind speed measurements. The NN setup with three hidden layers (eight neurons in the first, five in the second, and three in the third layer) is the same as for the other parameterizations without humidity.

In Fig. 8, in situ wind speeds are plotted against the wind speed resulting from marine radar images using the last GMF. The corresponding statistics of the comparison are given in the upper left of the scatterplots. The correlation coefficient is 0.99 , with a bias of $0.01 \mathrm{~m} \mathrm{~s}^{-1}$ and a standard deviation of $0.41 \mathrm{~m} \mathrm{~s}^{-1}$. The resulting parameterization statistically enables the retrieval of wind speeds as low as $\approx 0.75 \mathrm{~m} \mathrm{~s}^{-1}$.

\section{d. Discussion}

Overall, the sea surface stress is related to the mean wind speed at some reference level. The mean wind speed is thereby dependent on the sea state and the stratification conditions, given by the air-sea temperature difference and the relative air humidity. The sea state is characterized by the wave spectrum and its spectral and integral parameters. The mentioned investigations have shown that the main parameters are the SNR, which is proportional to $H_{S}^{2}$, and the peak wave phase speed $c_{p}$, which is determined from peak wave frequency $\omega_{p}$ and peak wavenumber $k_{p}$.

Table 2 gives a complete overview of comparisons of WiRAR wind speeds from each of the derived GMFs with in situ wind measurements. The first block summarizes all GMFs, considering only parameters determined from the radar datasets, as NRCS for the range- 


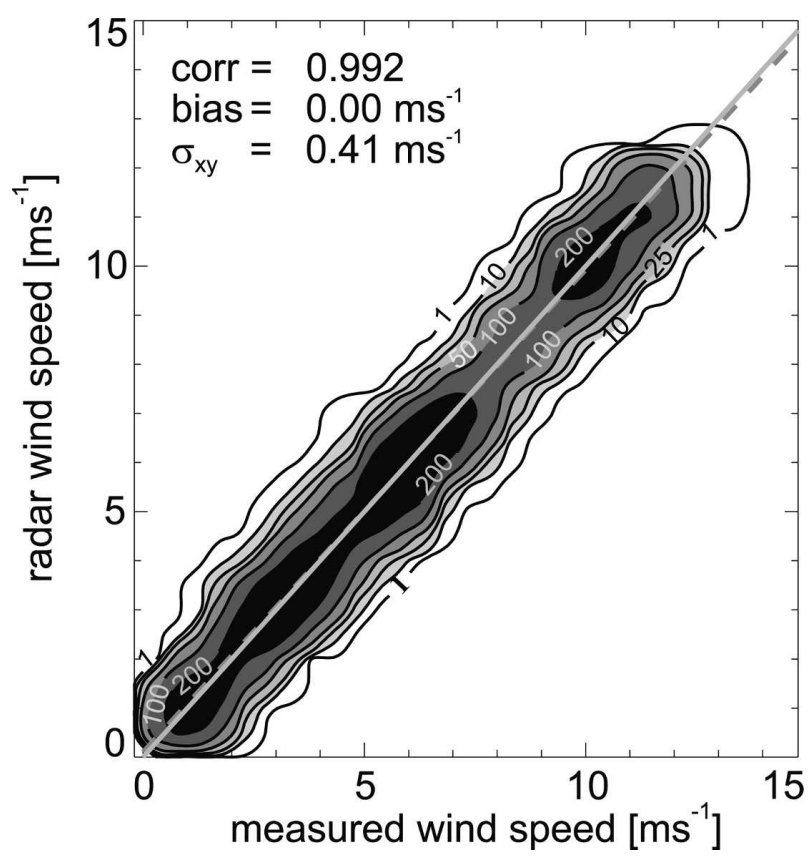

FIG. 8. Comparison of the wind anemometer wind speeds $u_{30}$ (mean of $10 \mathrm{~min}$ ) vs wind speeds retrieved from collocated marine radar images. The radar wind speeds were retrieved using an $\mathrm{NN}$ with the intensity of the mean radar image, antenna look direction, the wind direction from the collocated anemometer, SNR, air-sea temperature difference, and relative air humidity data as input (4786 datasets).

direction bins in crosswind directions, antenna viewing direction, wind direction, $c_{p}$, and/or SNR. Adding $c_{p}$ to the input-parameter list gives only a minor improvement in accuracy, whereas the SNR strongly improves the accuracy of the radar measurements. The second block shows an improvement in accuracy when the NNs incorporate external information about atmospheric conditions. The last block summarizes $\mathrm{NNs}$ using NRCS, sea state, and atmospheric information.

TABLE 2. Main statistical parameters resulting from the various GMFs considering the different input data.

\begin{tabular}{lccc}
\hline \hline \multicolumn{1}{c}{ Input parameters } & $\begin{array}{c}\text { Correlation } \\
\text { coefficient }\end{array}$ & $\begin{array}{c}\text { Bias } \\
\left(\mathrm{m} \mathrm{s}^{-1}\right)\end{array}$ & $\begin{array}{c}\sigma_{x y} \\
\left(\mathrm{~m} \mathrm{~s}^{-1}\right)\end{array}$ \\
\hline NRCS, $\Phi_{r}, \Phi_{w}$ & 0.959 & 0.01 & 0.90 \\
NRCS, $c_{p}, \Phi_{r}, \Phi_{w}$ & 0.955 & 0.00 & 0.86 \\
NRCS, SNR, $\Phi_{r}, \Phi_{w}$ & 0.971 & 0.00 & 0.76 \\
NRCS, $c_{p}, \operatorname{SNR}, \Phi_{r}, \Phi_{w}$ & 0.973 & 0.02 & 0.66 \\
NRCS, $q_{r}, \Phi_{r}, \Phi_{w}$ & 0.976 & 0.00 & 0.70 \\
NRCS $, \Delta \vartheta_{(a, s)}, \Phi_{r}, \Phi_{w}$ & 0.980 & 0.01 & 0.63 \\
NRCS, $q_{r}, \Delta \vartheta_{(a, s)}, \Phi_{r}, \Phi_{w}$ & 0.990 & 0.01 & 0.43 \\
NRCS, SNR, $\Delta \vartheta_{(a, s)}, \Phi_{r}, \Phi_{w}$ & 0.988 & 0.00 & 0.49 \\
NRCS, $c_{p}, \operatorname{SNR}, \Delta \vartheta_{(a, s)}, \Phi_{r}, \Phi_{w}$ & 0.987 & 0.01 & 0.47 \\
NRCS, SNR, $q_{r}, \Delta \vartheta_{(a, s)}, \Phi_{r}, \Phi_{w}$ & 0.992 & 0.00 & 0.41 \\
NRCS, $c_{p}, \operatorname{SNR}, q_{r}, \Delta \vartheta_{(a, s)}, \Phi_{r}, \Phi_{w}$ & 0.989 & 0.01 & 0.42 \\
\hline
\end{tabular}

Figure 9 shows the importance of sea state and atmospheric conditions when estimating wind speed. The bias (black curves) and standard deviation (gray curves) of the difference between radar and in situ wind speed are plotted against SNR (Fig. 9a), wave phase velocity at the spectral peak $c_{p}$ (Fig. 9b), air-sea temperature difference (Fig. 9c), and relative air humidity (Fig. 9d). The solid curves use the GMFs without considering any additional sea state and/or atmospheric parameters. The dashed curves show the resulting GMFs after adding the parameter indicated by the abscissa. Additionally, histograms (gray filled) of the available data are given.

Adding the SNR to the wind speed parameterization strongly improves the standard deviation for low SNRs. Further, the bias is decreasing for SNRs between 0.4 and 0.8. Taking $c_{p}$ into account for the GMF improves the standard deviation only slightly, while strongly decreasing the bias for $c_{p}$ between 6 and $8 \mathrm{~m} \mathrm{~s}^{-1}$. The slight effect of $c_{p}$ on the wind parameterization is explained with the given frequency distribution of $c_{p}$, which is concentrated between values of 8 and $12 \mathrm{~m} \mathrm{~s}^{-1}$. For this range the bias is about zero.

For the air-sea temperature difference the $\mathrm{NN}$ is under- and overestimating the wind speed for stable and unstable MABL, respectively. Especially under stable conditions the radar wind speeds are too low. Under equal wind speed conditions the sea surface friction and therefore the NRCS is lower for a stable MABL than for an unstable one. This is in agreement with theory and the observations of Keller et al. (1989). In the transition region the agreement is very good. Thus, accurate wind speed estimates require some knowledge of the atmospheric stability, which is given by the air-sea temperature difference. Considering the air-sea temperature difference results in an improvement of the bias and the standard deviation for both the unstable and stable conditions.

For low relative air humidity there is a high bias for the NN without considering humidity (solid curve). The $\mathrm{NN}$ is overestimating the wind speed, because the NRCS is larger for dryer air at the same stratification conditions and wind speed, for example, $u_{30}$. At higher relative air humidities the bias is lower, which is mainly caused by the higher number of datasets recorded under these conditions. Therefore, the additional knowledge of the air humidity, together with air-sea temperature difference and radar measurements, is important. Considering the relative air humidity as an additional atmospheric parameter results in an improvement of both bias and standard deviation for the whole humidity range (dashed curve).

In Fig. 10, the curves were retrieved using GMFs and 

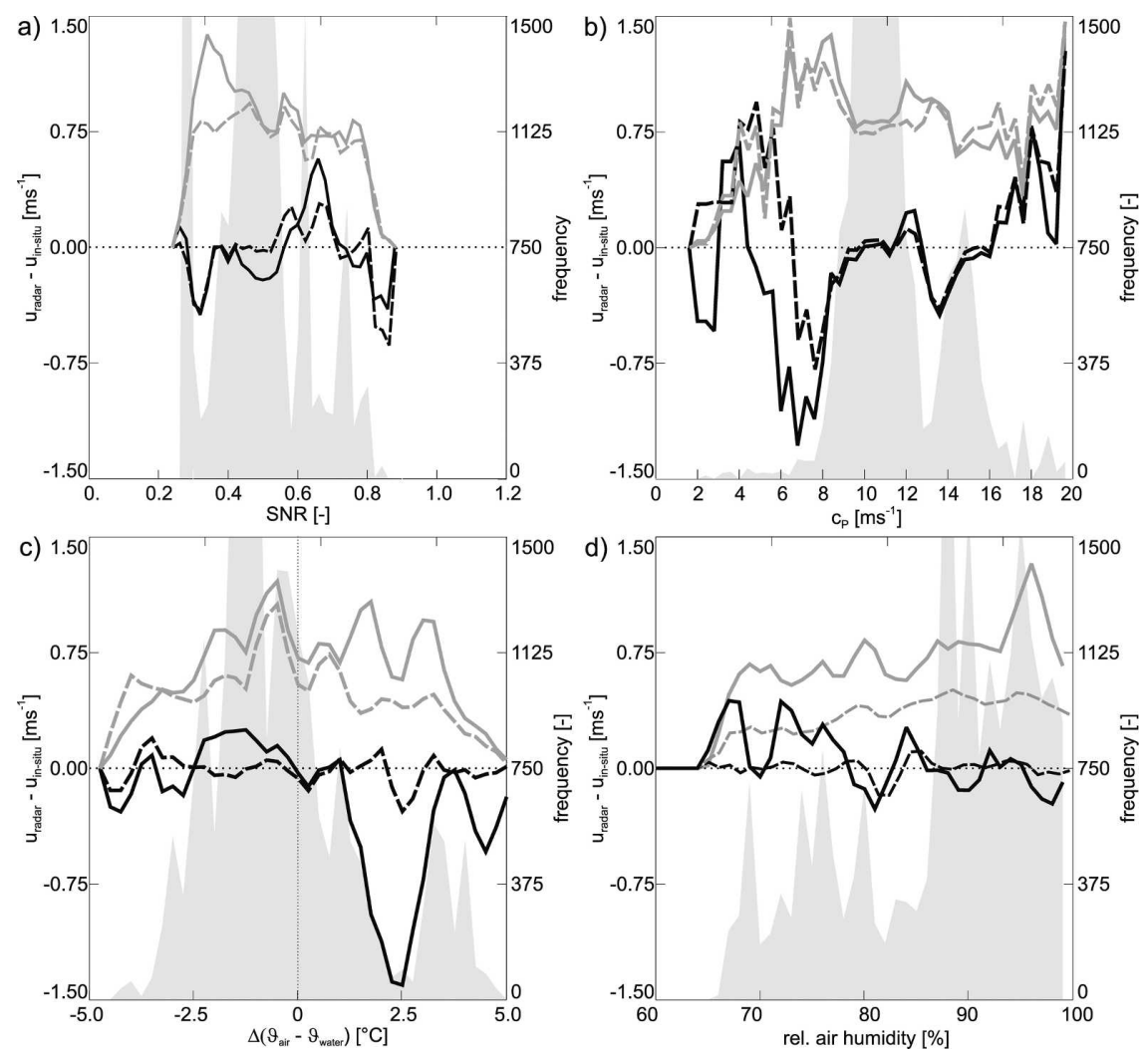

FIG. 9. Bias (black curves) and standard deviation (gray curves) of radar-retrieved wind speeds and in situ wind speeds over SNR, $c_{p}$, air-sea temperature difference, and relative air humidity. The solid curves give the parameterizations without considering additional sea state/atmospheric parameters. The dashed curves were retrieved using GMFs considering the $\mathrm{SNR}, c_{p}, \Delta \vartheta_{a, s}$, and relative air humidity. Histograms (gray filled) of all training/test datasets are plotted.

taking different additional parameters into account: only NRCS, antenna look direction, wind direction, and no additional sea state and/or atmospheric parameters (solid); additionally the SNR (long dashed); SNR and $c_{p}$ (short dashed); additionally only the air-sea temperature difference (dash-dot); and air-sea temperature difference and relative air humidity (dash-dotdot). The dotted curves give the result for the GMF taking all parameters into account.

The first GMF gives a bias changing from negative to higher positive values at wind speeds above $3 \mathrm{~m} \mathrm{~s}^{-1}$. The other parameterizations, which include a measure of stability, show a bias that is nearly zero up to $10 \mathrm{~m} \mathrm{~s}^{-1}$. All GMFs have a rather constant standard deviation. Above $10 \mathrm{~m} \mathrm{~s}^{-1}$ the bias increases significantly. The NNs are defined to force a total bias of zero. Due to the insufficient number of available data at higher wind speeds for the training of NNs, the bias is only very low for wind speeds below $10 \mathrm{~m} \mathrm{~s}^{-1}$. This problem is solvable by considering for the training of the $\mathrm{NN}$ a uniform wind speed distribution in the radar data.

\section{Conclusions and outlook}

A marine radar wind sensor, based on a new methodology for wind vector measurement called WiRAR, is described and validated with datasets from the FINO-I research platform in the German Bight. The system uses a marine $\mathrm{X}$-band radar as sensor with $\mathrm{HH}$ polarization. It is demonstrated that radar-image sequences of the ocean surface provide reliable information on ocean winds.

The marine X-band radar provides time series of radar backscatter images from the ocean surface. The radar technique thereby allows measurements under most weather conditions. In contrast to typical in situ sensors like anemometers, influences of the radar measurements by movements of ships or platforms and local turbulences induced by the platform installations are negligible. With the preexisting installations of radar systems on marine structures, harbors, platforms, and ships, the measurements can be acquired in a very cost-efficient way. 


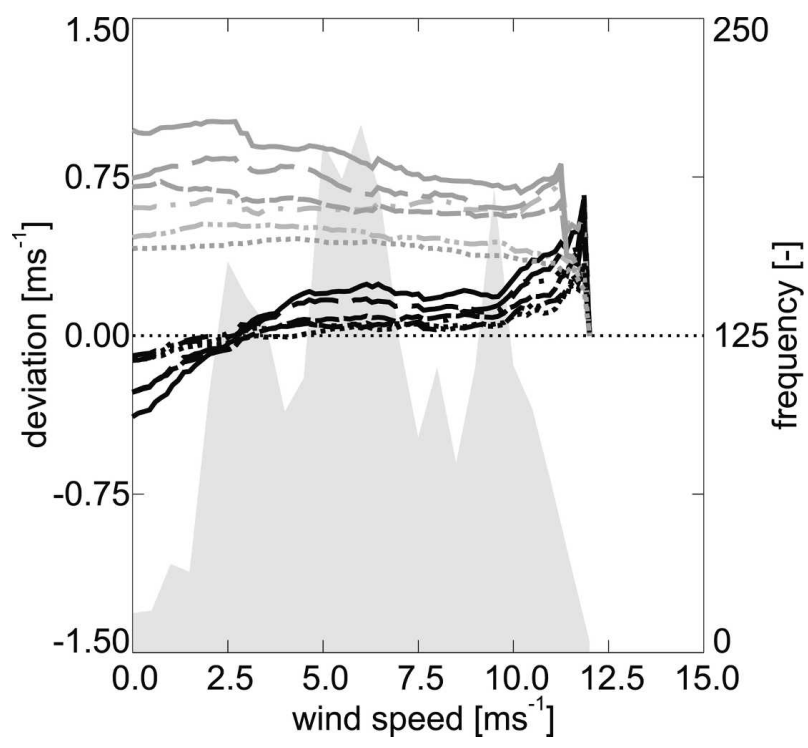

FIG. 10. Bias (black curve) and std dev (gray curve) in wind speed of comparisons of anemometer wind speeds to radar wind speeds for intervals of $0.2 \mathrm{~m} \mathrm{~s}^{-1}$. Radar wind speeds are determined without additional sea state or stratification parameters (solid curves), and considering additionally the sea state (dashed curves), the stratification conditions (dash-dot curves), or all parameters (dotted curve). In addition the histogram of wind speeds is plotted.

The NRCS of the ocean surface at X band with $\mathrm{HH}$ polarization and at grazing incidence is strongly dependent on the surface wind speed, wind direction, antenna look direction, and range distance, as well as on sea state and atmospheric conditions in the lower MABL. This provides the opportunity to develop an algorithm for remote measurements of surface wind vectors from radar images.

The algorithm, WiRAR, consists of two steps. In step one, wind directions are derived from wind-induced streaks, which are oriented in wind direction. This is done by determining the local gradients in the mean NRCS image, which give the orientation of the wind streaks, leaving a $180^{\circ}$ ambiguity. The ambiguity is removed by analyzing the movement of wind gust patterns in radar-image sequences. Comparison to in situ measurements resulted in a correlation of 0.99 , with a bias of $0.3^{\circ}$ and a standard deviation of $12.8^{\circ}$.

Wind speeds are retrieved in a second step from the dependence of the NRCS on wind speed and wind direction. This dependency is parameterized by training of an NN considering different input parameters, all retrieved from the radar datasets: the mean NRCSs in the crosswind direction at five different ranges (in the crosswind direction the wind field is not disturbed by the platform itself), the radar-retrieved wind direction, as well as the SNR and peak wave phase speed $c_{p}$, which provide information about the sea state. This gives already very good and practicable results with a correlation coefficient of 0.97 , a bias of $0.0 \mathrm{~m} \mathrm{~s}^{-1}$, and a standard deviation of $0.66 \mathrm{~m} \mathrm{~s}^{-1}$.

Wind speed estimates improve significantly when air-sea temperature is included as an additional input parameter. This is due to the additional dependence of the NRCS on the stability in the lower MABL. Comparison of radar-derived wind speeds (considering all these parameters) to in situ wind speeds measured at the platform at 30-m height resulted in a correlation of 0.99 , with a bias of $0.0 \mathrm{~m} \mathrm{~s}^{-1}$ and a standard deviation of $0.49 \mathrm{~m} \mathrm{~s}^{-1}$.

The air humidity, which is also provided at FINO-I along with many other meteorological parameters, has been taken into account as an additional parameter describing the atmospheric stability. The results could again be improved, resulting in a correlation of 0.99 , a bias of $0.0 \mathrm{~m} \mathrm{~s}^{-1}$, and a standard deviation of $0.41 \mathrm{~m} \mathrm{~s}^{-1}$.

The datasets investigated here were limited to wind speeds up to $\approx 16 \mathrm{~m} \mathrm{~s}^{-1}$. However, Hatten et al. (1998) have shown that for wind speeds up to $23 \mathrm{~m} \mathrm{~s}^{-1}$ no saturation in the NRCS occurs. Recently, more datasets from FINO-I are being received together with collocated meteorological in situ data, which will be considered for the purpose of covering a larger range of wind speeds in the WiRAR parameterization.

Because the radar system measures the wind-induced roughness at the ocean surface boundary layer, it in fact gives a measure of the wind-induced surface stress or momentum flux and therefore the friction velocity $u_{\star}$. Based on this, in a next step, WiRAR will be taken directly for measuring $u_{\star}$ (Horstmann and Dankert 2006). The main advantage is that no additional airwater-temperature or humidity measurements are required.

Acknowledgments. All radar-image sequences were kindly made available by the company Ocean-Waves (Lüneburg, Germany). The in situ data were kindly provided by the Bundesamt für Seeschifffahrt und Hydrographie (BSH) (Hamburg, Germany).

\section{REFERENCES}

Alpers, W., and K. Hasselmann, 1982: Spectral signal to clutter and thermal noise properties of ocean wave imaging synthetic aperture radars. Int. J. Remote Sens., 3, 423-446.

Askari, F., T. Donato, and J. Morrison, 1996: Detection of ocean wave fronts at low grazing angles using an X-band real aperture radar. J. Geophys. Res., 101, 20 883-20 898.

Bell, P., 1999: Shallow water bathymetry derived from an analysis of X-band marine radar images of waves. Coastal Eng., 37, 513-527.

Borge, J. N., K. Hessner, and K. Reichert, 1999: Estimation of the significant wave height with X-band nautical radars. Proc. 18th Int. Conf. on Offshore Mechanics and Arctic Engineering 
(OMAE'99), OMAE99/OSU-3063, St. John's, NL, Canada, American Society of Mechanical Engineers.

__ G. Rodríguez, K. Hessner, and P. González, 2004: Inversion of marine radar images for surface wave analysis. J. Atmos. Oceanic Technol., 21, 1291-1300.

Chaudhry, A., and R. Moore, 1984: Tower based backscatter measurements of the sea. IEEE J. Oceanic Eng., 9, 309-316.

Dankert, H., 2003: Retrieval of surface-current fields and bathymetries using radar-image sequences. Proc. Int. Geoscience and Remote Sensing Symp. (IGARSS'03), Toulouse, France, Geoscience and Remote Sensing Society, 2671-2673. , and W. Rosenthal, 2004: Ocean surface determination from X-band radar-image sequences. J. Geophys. Res., 109, C04016, doi:10.1029/2003JC002130.

_, J. Horstmann, S. Lehner, and W. Rosenthal, 2003a: Detection of wave groups in SAR images and radar-image sequences. IEEE Trans. Geosci. Remote Sens., 41, 1437-1446.

,$- \ldots$, and W. Rosenthal, 2003b: Ocean wind fields retrieved from radar-image sequences. J. Geophys. Res., 108, 3352, doi:10.1029/2003JC002056.

$\longrightarrow, \ldots$, and — 2004: Ocean surface winds retrieved from marine radar-image sequences. Proc. Int. Geoscience and Remote Sensing Symp. (IGARSS'04), Anchorage, AK, Geoscience and Remote Sensing Society, 1903-1906.

,-- , and - , 2005: Wind and wave field measurements using marine $\mathrm{X}$-band radar-image sequences. J. Oceanic Eng., 30, 534-542.

Donelan, M. A., 1990: Air-sea interaction. The Sea, B. LeMehaute and D. M. Hanes, Eds., Ocean Engineering Science, Vol. 9, Wiley and Sons, 239-292.

Drennan, W., H. Graber, D. Hauser, and C. Quentin, 2003: On the wave age dependence of wind stress over pure wind seas. J. Geophys. Res., 108, 8062, doi:10.1029/2000JC000715.

Drobinski, P., and R. Foster, 2003: On the origin of near-surface streaks in the neutrally-stratified planetary boundary layer. Bound.-Layer Meteor., 108, 247-256.

Geenaert, G., 1990: Measurements of the angle between the wind stress vector in the surface layer over the North Sea. J. Geophys. Res., 91, 7667-7679.

Hatten, H., F. Ziemer, J. Seemann, and J. Nieto-Borge, 1998: Correlation between the spectral background noise of a nautical radar and the wind vector. Proc. 17th Int. Conf. on Offshore Mechanics and Arctic Engineering (OMAE'98), Lisbon, Portugal, American Society of Mechanical Engineers.

Horstmann, J., and W. Koch, 2005: Measurement of ocean surface winds using synthetic aperture radars. J. Oceanic Eng., 30, 508-515.

— , and H. Dankert, 2006: Estimation of friction velocity using tower based marine radars. Proc. Int. Geoscience and Remote Sensing Symp. (IGARSS'06), Denver, CO, Geoscience and Remote Sensing Society, 1323-1326.

- W. Koch, S. Lehner, and R. Tonboe, 2002: Ocean winds from RADARSAT-1 ScanSAR. Can. J. Remote Sens., 28, 524-533.

- , H. Schiller, J. Schulz-Stellenfleth, and S. Lehner, 2003: Global wind speed retrieval from SAR. IEEE Trans. Geosci. Remote Sens., 41, 2277-2286.

—, D. Thompson, F. Monaldo, S. Iris, and H. Graber, 2005: Can synthetic aperture radars be used to estimate hurricane force winds? Geophys. Res. Lett., 32, 122801, doi:10.1029/ 2005GL023992.

Izquierdo, P., J. C. N. Borge, C. G. Soares, R. S. González, and G. R. Rodríguez, 2004: Comparison of wave spectra from nautical radar images and scalar buoy data. J. Waterw., Port, Coastal, Oceanic Eng., 131, 123-131.

Keller, W. C., W. J. Plant, and D. Weissman, 1985: The dependence of $\mathrm{X}$ band microwave sea return on atmospheric stability and sea state. J. Geophys. Res., 90, 1019-1029.

_ , V. Wismann, and W. Alpers, 1989: Tower-based measurements of the ocean C-band radar backscattering cross section. J. Geophys. Res., 94, 924-930.

$\longrightarrow,-$, R. A. Petitt, and E. A. Terray, 1994: Microwave backscatter from the sea: Modulation of received power and Doppler bandwith by long waves. J. Geophys. Res., 99, 97519766.

Koch, W., 2004: Directional analysis of SAR images aiming at wind direction. IEEE Trans. Geosci. Remote Sens., 42, 702710.

Komen, G., P. A. E. M. Janssen, V. Makin, and W. Oost, 1998: On the sea state dependence of the Charnock parameter. Global Atmos. Ocean Syst., 5, 367-388.

Lee, P., and Coauthors, 1995: X-band microwave backscattering from ocean waves. J. Geophys. Res., 100, 2591-2611.

_ J. Barter, E. Caponi, M. Caponi, C. Hindman, B. Lake, and H. Rungaldier, 1996: Wind-speed dependence of smallgrazing-angle microwave backscatter from sea surfaces. IEEE Trans. Antennas Propag., 44, 333-340.

Lyzenga, D., A. Maffet, and R. Shuchman, 1983: The contribution of wedge scattering to the radar cross section of the ocean surface. IEEE Trans. Geosci. Remote Sens., GE-21, 502-505.

Phillips, O. M., 1977: Dynamics of the Upper Ocean. 2d ed. Cambridge University Press, $336 \mathrm{pp}$.

Plant, W. J., 1989: The modulation transfer function, concept and applications. Radar Scattering from Modulated Wind Waves, G. J. Komen and W. A. Oost, Eds., Kluwer Academic, 155172.

Richaume, P., F. Badran, M. Crepon, C. Mejia, H. Roquet, and S. Thiria, 2000: Neural network wind retrieval from ERS-1 scatterometer data. J. Geophys. Res., 105, 8737-8751.

Senet, C., J. Seemann, and F. Ziemer, 2001: The near-surface current velocity determined from image sequences of the sea surface. IEEE Trans. Geosci. Remote Sens., 39, 492-505.

Stewart, R., 1974: The air-sea momentum exchange. Bound.Layer Meteor., 6, 151-167.

Stoker, J., 1957: Water Waves: The Mathematical Theory with Applications. Wiley-InterScience, $567 \mathrm{pp}$.

Trizna, D., 1997: A model for Brewster angle effects on sea surface illumination for sea scatter studies. IEEE Trans. Geosci. Remote Sens., 35, 1232-1244.

_ 2001: Errors in bathymetric retrievals using linear dispersion in 3-d FFT analysis of marine radar ocean wave imagery. IEEE Trans. Geosci. Remote Sens., 39, 2465-2469.

— angle radar sea scatter in nearshore regions. IEEE Trans. Geosci. Remote Sens., 34, 747-757.

Wetzel, L., 1990: Electromagnetic scattering from the sea at low grazing angles. Surface Waves and Fluxes, G. L. Geernaert and W. L. Plant, Eds., Vol. 2, Remote Sensing, Kluwer Academic, 109-171.

Young, I., W. Rosenthal, and F. Ziemer, 1985: A threedimensional analysis of marine radar images for the determination of ocean wave directionality and surface currents. $J$. Geophys. Res., 90, 1049-1059.

Ziemer, F., 1991: Directional spectra from shipboard navigation radar during LEWEX. Directional Ocean Wave Spectra, R. C. Beal, Ed., The Johns Hopkins University Press, 80-84. 